\title{
TOTEM Physics plans
}

\section{Mirko Berretti ${ }^{* \dagger}$}

INFN Pisa \& University of Siena, Department of Physics, Via Roma, 56 - 53100 Siena, Italy.

E-mail: Mirko.Berrettiecern.ch

The TOTEM experiment at the LHC is designed and optimized to measure the total proton-proton cross section with a precision of $1 \div 2 \%$, to study the nuclear elastic proton-proton cross section over a wide range of the squared four-momentum transfer and to perform a comprehensive physics program on diffractive processes partially in cooperation with the CMS experiment. In this contribution the status and the Physics program of the TOTEM experiment will be presented as well as the prospects for the TOTEM standalone measurements at the startup of the LHC.

European Physical Society Europhysics Conference on High Energy Physics, EPS-HEP 2009, July 16 - 222009

Krakow, Poland

\footnotetext{
* Speaker.

$\dagger$ On Behalf of the TOTEM Collaboration.
} 


\section{Introduction}

The TOTEM experiment [1] is dedicated to the precise measurement of the total proton-proton cross-section $\left(\sigma_{t o t}\right)$ using the luminosity-independent method, based on the Optical Theorem. A $\sigma_{t o t}$ uncertainty of $1 \div 2 \%$ can be reached by measuring the elastic scattering at low fourmomentum transfer $\left(|t| \sim p^{2} \theta^{2} \sim 10^{-3} \mathrm{GeV}^{2}\right)$ as well as the inelastic interaction rate with losses reduced to few per-cents. Furthermore, TOTEM aims at a deeper understanding of the proton structure by measuring the central elastic scattering $\left(|t|>1 \mathrm{GeV}^{2}\right)$ and by performing a comprehensive study of diffraction, spanning from cross-section measurements of individual diffractive processes to the analysis of their event topologies. A flexible trigger provided by its detectors will allow to take data under all LHC running scenarios. The combination of the CMS [2] and TOTEM experiments gives the opportunity to study a wider range of diffractive processes, including hard diffraction, with an unprecedented coverage in rapidity. For this purpose the TOTEM trigger and data acquisition (DAQ) systems are designed to be compatible with the CMS ones, in order to allow common data taking periods at a later stage [3]. In the following, after a general overview of the experimental apparatus, the main features of the TOTEM physics program will be described as well as the measurements planned for the first year of LHC operation.

\section{The TOTEM experimental apparatus}

TOTEM is composed by three detectors, placed symmetrically around the Interaction Point (IP) 5 of the LHC, shared with the CMS experiment: the T1 and T2 telescopes and the Roman Pot (RP) stations. T1 and T2, placed at about 10 and $14 \mathrm{~m}$ from the IP, reconstruct the charged particle tracks produced by inelastic processes in the pseudorapidity $(\eta)$ range $3.1<|\eta|<4.7$ (T1) and $5.3<|\eta|<6.5$ (T2); the Roman Pot stations, equipped with silicon detectors, are placed at 147 and $220 \mathrm{~m}$ from the IP and measure elastically and diffractly scattered protons with scattering angles of a few to hundred $\mu \mathrm{rad}$. Since T1 and T2 are dedicated to the measurement of the inelastic rate, they also reconstruct the primary vertex in order to distinguish between beam-beam events and background. Moreover, thanks to their good pseudorapidity resolution, T1 and T2 are also used for forward charged particle multiplicity studies. Both telescopes are gas detectors, in particular T1 is made by 5 planes of CSC while T2 is based on triple GEM detectors [4]. Their track $\eta$-resolution $(\Delta \eta)$, which depends on the particle energy and $\eta$, is $0.02<\Delta \eta<0.2$ and $0.04<\Delta \eta<0.1$ respectively for T1 and T2. One or maybe two T1 arms will be installed in September 2009 while both T2 arms are already installed. Protons are detected by micro-strip silicon detectors placed inside a secondary vacuum vessel (called Pot) inserted in the beam pipe. The Pot can then be moved as close as possible to the beam allowing to detect small angle protons with an acceptance strongly dependent on the LHC optics (see section 3 and 4 ). Safety requirements, which don't allow the detectors to stay closer than $10 \sigma_{\text {Beam }}$ from the beam axis, have driven TOTEM to develop a new detector edge technology [5] which reduces the dead area at the edge to only $50 \mu \mathrm{m}$. This improvement is a crucial aspect of the experiment, as it makes possible to reach the small $|t|$ elastic scattering necessary for a precise $\sigma_{t o t}$ measurement. The installation of RP $220 \mathrm{~m}$ stations has been completed in July 2009. The read-out of all TOTEM sub-detectors is based on the digital VFAT chip [6], specifically designed for TOTEM and characterized by trigger capabilities. 


\section{TOTEM Physics program}

TOTEM is dedicated to the $\sigma_{t o t}$ measurement with an ultimate precision of $1 \div 2 \%$, to the differential elastic cross section $\left(\frac{d \sigma_{e l}}{d t}\right)$ measurement over a wide range of $|t|\left(2 \cdot 10^{-3}<|t|<10 \mathrm{GeV}^{2}\right)$ and to the study of the inelastic interaction by measuring the cross section of soft diffractive processes and the forward charged particle flows. TOTEM can perform this program operating stand alone while the cooperation with CMS makes possible to study hard diffraction, low-x Physics, central exclusive diffractive production and to combine the particle and energy flows even in the forward region [3]. The best fit performed by the COMPETE collaboration [7] using all the available pp and $\mathrm{p} \overline{\mathrm{p}}$ data, has estimated $\sigma_{\text {tot }}=111.5 \pm 1.2_{-2.1}^{+4.1} \mathrm{mb}$ at the LHC energy. However different models of the hadronic cross section, which cannot be excluded because of the Tevatron discrepancy and the large error in the high energy cosmic ray data, predict the $\sigma_{t o t}$ value in a range from 90 to 130 mb. The TOTEM $\sigma_{\text {tot }}$ measurement with $1 \div 2 \mathrm{mb}$ error allows to distinguish among these different models. To measure $\sigma_{t o t}$ and the LHC luminosity $(\mathscr{L})$ TOTEM will use the luminosity independent method based on the Optical Theorem:

$$
\sigma_{\text {tot }}=\frac{16 \pi}{1+\rho^{2}} \frac{d N_{e l} /\left.d t\right|_{t=0}}{N_{e l}+N_{\text {inel }}} \quad \mathscr{L}=\frac{1+\rho^{2}}{16 \pi} \cdot \frac{\left(N_{e l}+N_{\text {inel }}\right)^{2}}{d N_{e l} /\left.d t\right|_{t=0}}
$$

The quantities in eq. 3.1 measured by TOTEM are: the inelastic rate $N_{\text {inel }}$, the elastic rate $N_{e l}$ and the extrapolated value of $\frac{d N e l}{d t}$ to $t=0$. The $\rho$ parameter $\left(\rho=\left.\frac{\Re\left(A_{e l}\right)}{\mathfrak{I}\left(A_{e l}\right)}\right|_{t=0}, A_{e l}\right.$ being the nuclear elastic amplitude) is extrapolated from existing data. The acceptance in and measurement of the proton kinematical parameters strongly depends from the LHC optics. The smaller the betatron function at the IP $\left(\beta^{*}\right)$, the bigger the beam divergence and the uncertainty on the proton scattering angle. Therefore TOTEM needs high $\beta^{*}$ optics to measure the small proton scattering angle for the elastic rate extrapolation (see fig. 1, left). With a $\beta^{*}=1540 \mathrm{~m}$ optics TOTEM will measure the elastic rate in the range $0.002<|t|<0.2$ where $\frac{d \sigma_{e l}}{d t}$ is approximately described by an exponential distribution; the elastic rate will be then extrapolate to $t=0$. With a $\beta^{*}=90 \mathrm{~m}$ optics the RP acceptance starts from $|t|=0.02$ allowing a less precise extrapolation. Both optics allow TOTEM to perform the $\sigma_{t o t}$ measurement in about one day of data acquisition, considering a luminosity of $\sim 10^{28}$ and $10^{30} \mathrm{~cm}^{-2} \mathrm{~s}^{-1}$ respectively for $\beta^{*}=1540 \mathrm{~m}$ and $\beta^{*}=90 \mathrm{~m}$ optics. Small $\beta^{*}$ optics, for which the RP $|\mathrm{t}|$-acceptance starts at higher $|\mathrm{t}|$-values, are important for the test of different cross section models describing the central elastic interaction. Concerning the inelastic rate measurement, the most important inelastic trigger inefficiency is given by losses of low mass Single Diffractive (SD) events. The relative uncertainty of the inelastic rate measurement (including an estimate of the rate outside the TOTEM acceptance obtained using an extrapolation based on the measured rate within the TOTEM acceptance) is estimated to be about $1 \%$. This is the most significant experimental uncertainty on the $\sigma_{t o t}$ measurement for $\beta^{*}=1540 \mathrm{~m}$ runs, while if $\beta^{*}=90 \mathrm{~m}$, the biggest uncertainty is the error on the extrapolation of the elastic distribution to $t=0$ (4\%). For both optics the error on the $\rho$ parameter has been taken into account (1.2\%). The relative error on $\sigma_{\text {tot }}$ including all the contributions is expected to be about $1 \div 2 \%$ and $\sim 5 \%$ for the $\beta^{*}=1540$ and $90 \mathrm{~m}$ optics respectively. The diffractive proton acceptance for the RP at $220 \mathrm{~m}$ has been computed for various optics as a function of $\xi$ and $t$ (fig. 1, right) as well as the Double Pomeron Exchange (DPE) central mass $(M)$ acceptance. By combining data from low, intermediate and high $\beta^{*}$ runs, the differential cross-section as a function of $M$ can be measured with good precision over the full mass range. 

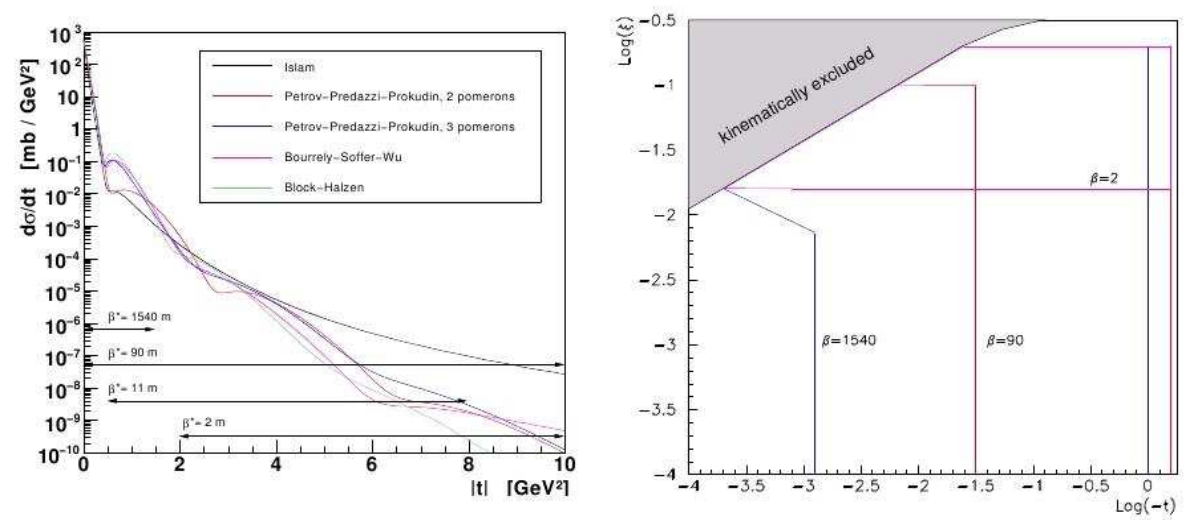

Figure 1: Left: $\frac{d \sigma_{e l}}{d t}$ at $\sqrt{s}=14 \mathrm{TeV}$ as predicted by various models together with the t-acceptance ranges for different optics configurations. Right: contour lines of $10 \%$ acceptance for RP220 in $\log _{10}|t| \operatorname{and}_{10} \log _{10} \xi(\xi$ is the fractional proton momentum loss) for diffractively scattered protons at different optics configurations.

\section{Early measurements}

In the first year of the LHC operation a reduced c.m. energy and low $\beta^{*}$ are scheduled. With low luminosity TOTEM has the opportunity to make first soft diffractive studies in stand-alone runs. The acceptance of the diffractive protons obtained at $\sqrt{s}=10 \mathrm{TeV}$ and $\beta^{*}=3 \mathrm{~m}$ is quite similar to the corresponding $\sqrt{s}=14 \mathrm{TeV}$ case. In these conditions, diffractive cross section can be measured in the $0.02<\xi<0.18$ and $2<|t|<10 \mathrm{GeV}^{2}$ ranges, the latter being also the allowed t-range for the elastic cross section measurement. Consequently, the DPE central mass that can be reconstructed with this optics is $0.25<M<1.8 \mathrm{TeV}$. Using the T1 and T2 tracks, it is possible to compare the $\xi$ measured by RP with the rapidity gap $\Delta \eta$ obtained using also the inelastic telescopes (for example that the relation $\Delta \eta=-\ln \xi$ holds in SD events). The measurements of forward charged multiplicity at LHC energies is also important for interpretation of cosmic ray (CR) air shower data: the small CR flux around $100 \mathrm{PeV}$ makes difficult to tune on data the Monte Carlo which simulate the air-showers and discrepancies between models have been found, as for example in the prediction of forward charged multiplicity. Studying the pp collisions at the LHC the $100 \mathrm{PeV} \mathrm{CR}$ interaction can be investigated and TOTEM, by measuring the forward charged particle flow, can provide an important contribution to the tuning of hadronic showers.

\section{References}

[1] TOTEM TDR, [CERN-LHCC-2004-002]; addendum [CERN-LHCC-2004-020] (2004).

[2] CMS Physics TDR, Volume I, [CERN-LHCC-2006-001] (2006).

[3] CMS and TOTEM Collaborations, [CERN-LHCC-2006-039/G-124] (2006).

[4] F. Sauli, Nucl. Instrum. Methods A 386, 531 (1997).

[5] E. Noschis et al., Nucl. Instrum. Methods A 563, 41 (2006).

[6] G. Anelli et al. (TOTEM Collaboration), JINST 3:S08007 (2008).

[7] J.R. Cudell et al. (COMPETE Collaboration), Phys. Rev. Lett. 89, 201801 (2002). 